\title{
O uso de Veículos Aéreos Não Tripulados (VANTs) nas operações de paz da ONU: o caso da MINUSMA
}

\author{
The use of Unmanned Aerial Vehicles (UAVS) in the \\ UN peace operations: the case of MINUSMA
}

DOI: $10.21530 /$ ci.v15n2.2020.1005

Sérgio Luiz Cruz Aguilar ${ }^{1}$

Jéssica Tauane dos Santos²

\section{Resumo}

Em 2015, a Organização das Nações Unidas (ONU) introduziu Veículos Aéreos Não Tripulados (VANTs) na Missão Multidimensional Integrada de Estabilização das Nações Unidas no Mali (MINUSMA). Este trabalho utiliza o caso da MINUSMA para discutir o uso dessa tecnologia nas operações de paz. O argumento central é que a utilização de VANTs no Mali é fruto de inovação adaptativa no campo das missões de paz. Para substanciar o argumento, utilizam-se documentos da ONU, fontes secundárias e entrevistas. A análise utiliza elementos selecionados da chamada 'revolução de assuntos militares' e da literatura sobre inovação militar: doutrina, organização, custos, domínio da informação, comando e controle, simulação e treinamento, e agilidade/ímpeto. O texto apresenta alguns aspectos da 'revolução de assuntos militares' e da inovação militar, descreve a evolução nas operações de paz que resultou no uso de VANTs e o contexto em que eles foram desdobrados no Mali para, no final, discutir a utilização dessa tecnologia na MINUSMA e nas missões de paz em geral.

Palavras-chave: ONU; Operações de Paz; VANTs; MINUSMA; Inovação Militar.

1 Livre Docente em Segurança Internacional pela UNESP. Pós-doutorado em Segurança Internacional na Universidade de Oxford - Reino Unido. Professor do Departamento de Sociologia e Antropologia da UNESP — Campus de Marília/SP e dos programas de pós-graduação San Tiago Dantas em Relações Internacionais (UNESP/UNICAMP/ PUC-SP) e em Ciências Sociais (UNESP - Campus de Marília/SP). Bolsista de produtividade em pesquisa do CNPq. ORCID: http://orcid.org/0000-0003-4757-4426; email: sergio.aguilar@unesp.br.

2 Graduada em Relações Internacionais pela UNESP-Campus de Marília/SP. Mestranda em Relações Internacionais no Programa San Tiago Dantas (UNESP-UNICAMP-PUC/SP). Membro do Grupo de Estudos e Pesquisa em Conflitos Internacionais (GEPCI) e do Grupo de Estudos de Defesa e Segurança Internacional (GEDES). Foi bolsista de Iniciação Científica da Fundação de Amparo à Pesquisa do Estado de São Paulo (FAPESP). Bolsista da CAPES. ORCID: https://orcid.org/0000-0003-2888-6547; email:jessica.santos@unesp.br.

Artigo submetido em 17/09/2019 e aprovado em 15/03/2020. 


\begin{abstract}
In 2015, the United Nations (UN) introduced Unmanned Aerial Vehicles (UAVs) into the Multidimensional Integrated Stabilization Mission in Mali (MINUSMA). This article uses the case of MINUSMA to discuss the use of this technology in peacekeeping operations. The central argument is that the use of UAVs in Mali is result of an adaptive innovation in the field of peace operations. The argument is substantiated through UN documents, secondary sources and interviews. The analysis is based on selected elements from the revolution in military affairs and military innovation literature: doctrine, organization, costs, domain of information, command and control, simulation and training, and agility/impetus. The article presents some aspects of the revolution in military affairs and military innovation, describes an evolution of peacekeeping that led to the use of UAVs and the context in which they were deployed in Mali to discuss, in the last part, the use of such technology in MINUSMA and peace operations in general.
\end{abstract}

Keywords: UN; Peacekeeping Operations; UAVs; MINUSMA; Military Innovation.

\title{
Introdução
}

Diante da complexidade das missões de paz multidimensionais³ ${ }^{3}$ a Organização das Nações Unidas (ONU) tem buscado novas formas de responder aos desafios impostos por essas missões no terreno, seja implementando modificações institucionais, tanto no nível estratégico quanto operacional, seja utilizando novas tecnologias4. A introdução dos Veículos Aéreos Não Tripulados (VANTs) em algumas de suas operações de paz, com fins de vigilância, desde 2013, é um exemplo dessas mudanças. ${ }^{5}$

3 Operações integradas por diversos componentes, incluindo militares, policiais e civis que atuam em diversas áreas como assuntos políticos, Estado de Direito, direitos humanos, reconstrução, assuntos humanitários, gênero, etc. (UN, 2003).

4 Entendidas como algo que amplia nossas capacidades (Rutherford e Ahlgren 1991) ou provedoras de determinada funcionalidade (Arthur 2009).

5 Em inglês Unmanned Aerial Vehicles (UAV) ou Unmanned Aerial Systems (UAS). De forma geral, os termos drone, UAS e UAV vem sendo utilizados como sinônimos, especialmente no meio midiático, todavia, o termo UAS é considerado mais completo por englobar todos os aspectos do desdobramento desses veículos, como a estação de controle, comunicações, equipamentos de apoio etc. e não apenas o veículo (What is the Difference 2016). A nomenclatura foi, então, atualizada para abranger todo o sistema - estação em solo e enlace de pilotagem com outros componentes para a realização do voo - sendo denominado em inglês Remotely Piloted Aircraft System (RPAS), em português Sistema Aéreo Remotamente Pilotado (SARP) (Magella 2016). No entanto, a maior parte da bibliografia consultada e a resolução do Conselho de Segurança da ONU, que autorizou o uso dessa tecnologia na República Democrática do Congo, utilizaram o termo UAS. Dessa forma, apesar da tendência ser no sentido de utilizar-se o termo SARP, nesse texto utilizaremos o termo VANTs como sinônimo de UAV, UAS ou RPAS. Utilizamos, também, a palavra drones no mesmo sentido. 
O primeiro emprego de VANTs em uma operação de paz da ONU ocorreu na República Democrática do Congo (RDC), na MONUSCO, e foi considerado pela própria missão como benéfico para identificar movimentos de grupos armados, monitorar campos de desalojados, reconhecimento em vastas áreas e melhorar a capacidade de alerta antecipado e o conhecimento situacional no terreno (UN 2013b). A partir dessa experiência, a ONU introduziu a tecnologia também na Multidimensional Integrated Stabilization Mission in Mali (MINUSMA) em 2015 (UN Flying Hermes 2016).

O uso militar dos VANTs ocorreu como parte da chamada 'revolução dos assuntos militares' (RAM), iniciada nos Estados Unidos (EUA) na década de 1990 (PERON, 2016). Um dos propósitos da RAM foi adaptar as missões aos novos ambientes conflitivos em que elas passaram a ser desdobradas, de modo a dotá-las de maior capacidade de fazer frente às novas demandas que surgiram. Nas missões de paz, argumentamos que o uso de VANTs ocorreu no mesmo sentido.

Dessa forma, o texto utiliza o caso da MINUSMA para discutir o uso de VANTs nas operações de paz. O argumento central é que a utilização de VANTs no Mali é fruto de inovação adaptativa no campo das missões de paz. O estudo de caso utiliza documentos da ONU, fontes secundárias e entrevistas. A análise é baseada em elementos da RAM e da literatura sobre inovação militar, selecionados pelos autores: doutrina, organização, custos, domínio da informação, comando e controle, simulação e treinamento, e agilidade/ímpeto.

O texto inicia-se com alguns aspectos da 'revolução de assuntos militares' e da inovação militar. Em seguida, descreve a evolução nas operações de paz que resultou no uso de VANTs e o contexto em que eles foram desdobrados no Mali. No final, discute a utilização dessa tecnologia na MINUSMA e nas missões de paz em geral.

\section{Revolução, inovação, adaptação dos meios militares}

A história é repleta de exemplos do impacto de tecnologias sobre as práticas da guerra, de tecnologias militares que foram utilizadas na vida civil e tecnologias civis que aumentaram as capacidades militares, da mesma forma que 'revoluções' em assuntos militares que modificaram a condução da guerra.

Com o fim da bipolaridade da Guerra Fria, novas formas de conflito surgiram com a presença de atores não estatais impactando a segurança em Estados incapazes 
ou pouco dispostos a cumprir com suas obrigações. Os conflitos intraestatais assimétricos, envolvendo grupos não estatais (insurgentes, terroristas, milícias, organizações criminosas e outros) com objetivos militares e políticos divergentes e meios desproporcionais, tornam-se recorrentes. Na busca por vantagens estratégicas e táticas, incluindo o controle coercitivo sobre populações locais, esses grupos vitimam civis de forma recorrente o que, em alguns casos, acontece também como prática das forças de segurança estatais (Wood, Kathman e Gent 2012; Valentino, Huth e Balch-Lindsay 2004). As operações irregulares praticadas por esses grupos, resultaram na chamada guerra híbrida, que mistura táticas regulares e irregulares, armamentos convencionais e não convencionais, atos terroristas, combatentes misturando-se a populações civis, gerando um ambiente complexo e prolongando os conflitos (Arreguín-Toft 2005; Deshpande 2018; Fridman 2018).

Nesse contexto, as intervenções internacionais tiveram que se adaptar a essas novas dinâmicas dos conflitos. Nos EUA, parte dessa adaptação envolveu a chamada 'revolution in military affairs' (RMA), conduzida a partir da década de 1990 para reforma de suas forças armadas de modo a consolidar a sua proeminência no globo e fazer frente às novas demandas que surgiram após o fim da Guerra Fria. A RMA se relacionava com a "redução iminente das dimensões das forças armadas [...] e a sustentação de uma estrutura de capacidades combatentes elevadas em um ambiente de incerteza e de ambiguidades de cenários de ameaça e confronto” (Duarte 2012, 9). A 'revolução’ foi centrada na inovação tecnológica (sistemas) que garantia o domínio informacional (processos) necessário para diminuir as incertezas e ambiguidades das operações militares. A RMA envolveu, então, o desenvolvimento de sistemas de armamentos e de processos mais eficientes de manutenção de tropas em operações e a exploração dos efeitos táticos e estratégicos da tecnologia militar (Cohen 2004; Duarte 2012).

Concordamos que tecnologia não é sinônimo de inovação, apesar de inovações normalmente envolverem tecnologia. Notoriamente, é difícil definir o significado de inovação. Os estudiosos têm problemas em tal definição e, por vezes, não a diferenciam de outras mudanças ou apresentam conceitos contraditórios. No entanto, a partir da reflexão sobre os vários estudos existentes, Grissom (2006) salientou que três atributos constituiriam uma definição consensual de inovação militar: ela muda a maneira pela qual as formações militares funcionam no terreno; é significativa em escopo e impacto; e é tacitamente equiparada com maior eficácia militar. 
Farrell, Rynning e Terriff (2013) afirmaram que as organizações militares reagem positivamente às inovações tecnológicas e operacionais se a inovação "melhora os modos de guerra tradicionalmente valorizados [...], busca melhorar os modos de guerra subvalorizados ou desenvolver formas totalmente novas de guerra" (Farrell, Rynning e Terriff 2013, 8, tradução nossa). ${ }^{6}$ Enquanto a chamada inovação adaptativa aumenta a eficácia/eficiência da execução de tarefas tradicionais, a inovação disruptiva desafia as tarefas principais da organização e exige mudanças mais profundas. Dessa forma, a inovação adaptativa é mais provável de ocorrer do que a disruptiva.

O Almirante Wylie trabalhou as mudanças no campo de batalha, no mar, ou na área política sobre a guerra e usou a palavra adaptação, que engloba 'movimentos adaptativos' que as partes fazem em relação as outras, sejam eles tecnológicos, táticos, materiais ou analíticos, como um processo interativo de ciclos de feedback. Essa adaptação recíproca é utilizada pelos beligerantes para melhorar sua posição em relação ao adversário ou ao status quo ante (Wylie Jr 1989). Pode-se entender o termo 'adaptação' por ele utilizado com o sentido de inovação.

Inovação pode ser conceituada como "um processo pelo qual as empresas dominam e implementam o design e a produção de bens e serviços novos para elas, independentemente de serem ou não novos para seus concorrentes" (Ernst, Mytelka e Ganiatsos 1998 apud Mytelka 2000, 18, tradução nossa). ${ }^{7}$ A autora tratou da inovação como sistema que emerge através de múltiplos atores, suas interações e aprendizados. Nesse sentido, "o escopo da inovação não inclui apenas tecnologia e produção, mas organizações (no sentido de atitudes, práticas e novas formas de trabalho)". O conceito de sistemas de inovação enfatiza a adaptação como elemento central da capacidade de inovação, ou seja, inovação como capacidade de se adaptar a mudanças (World Bank 2006).

Um estudo do Center for Strategic and International Studies, de 1993, apresentou a revolução técnica militar sob o ponto de vista de nove elementos: o chamado 'quadro integrativo', envolvendo doutrina e organização; os 'habilitadores de capacidades', incluindo domínio da informação, comando e controle, simulação e treinamento, e agilidade; e 'capacidades de execução', que abarcaram armas inteligentes, plataformas e armas exóticas (Mazzar, Schaffer e Ederington 1993). Os autores salientaram que a verdadeira RMA é obtida por meio da combinação

6 "[...] improve on traditionally valued ways of war [...], seeks to improve undervalued ways of war, or to develop wholly new ways of war".

7 "[...] the process by which firms master and implement the design and production of goods and services that are new to them, irrespective of whether or not they are new to their competitors". 
de tecnologia, organização e doutrina. A falta de uma delas indicaria uma evolução, ao invés de 'revolução'. Além disso, a literatura sobre inovação militar apresenta que a extensão da incorporação de novas tecnologias nas organizações depende: dos custos financeiros da inovação; do ímpeto pela inovação; e do grau da mudança (Rosen 1991; Farrell, Rynning e Terriff 2013; Schaub Jr 2015). Dessa forma, utilizaremos os elementos doutrina, organização, custos, domínio da informação, comando e controle, simulação e treinamento, e agilidade/ímpeto para nortear as discussões sobre o emprego de VANTs.

\section{O uso de VANTs nas operações de paz}

Os VANTs são entendidos como "pequenos aviões que voam por controle remoto ou de forma autônoma"8 (Gilman 2014, 2, tradução nossa). A ideia do que seria um veículo sem tripulação surgiu dos balões armados austríacos, utilizados a partir de 1849 (Diálogo Setorial União Europeia-Brasil 2017). Em 1916, os norteamericanos testaram o Aerial Torpedo que, por falta de maturidade técnica, não foi empregado em nenhuma das grandes guerras (Oliveira 2016; Nonami et al 2010). A Alemanha utilizou as chamadas bombas voadoras do tipo V-1 e V-2 contra o Reino Unido com o objetivo de atacar alvos a partir de grandes distâncias sem utilizar pilotos (Chamayou 2015; Oliveira 2016). Entre as décadas de 1950 e 1970, a evolução da pesquisa permitiu a construção do Ryan Model 147A Fire Fly, equipado com câmeras e empregado para tirar fotos durante a Guerra do Vietnã, utilizadas para análise das atividades do inimigo (Cooke 2017; Chamayou 2015). Com o final daquela guerra, os EUA não se interessaram em dar continuidade ao desenvolvimento de drones. Israel, entretanto, herdou algumas dessas máquinas e as utilizou em 1973, na Guerra do Yom Kippur e, posteriormente, em 1982, na planície do Bekaa contra os sírios. Nas duas situações, os drones foram usados para atrair fogo inimigo e, em seguida, o exército israelense atacou pontos estratégicos localizados pelas câmeras desses equipamentos (Chamayou 2015).

Na Guerra do Golfo (1991), os EUA utilizaram o Pioneer, drone desenvolvido por Israel, em missões de vigilância (Cole 2014). Posteriormente, em 1999, o Predator (predador em português), novo protótipo estadunidense, foi utilizado no Kosovo para filmar e indicar alvos com laser para que os aviões F16 atacassem. Esse modelo só foi equipado com mísseis em 2001 e, ao final do mesmo ano,

8 "[...] small aircraft that fly by remote control or autonomously." 
foi utilizado para ataques no Afeganistão (Chamayou 2015). Houve, então, uma escalada no uso de drones. Em 2015, mais de 90 países utilizavam drones para tarefas de reconhecimento, coleta de dados de inteligência e targeting ${ }^{9}$ (West e Bowman 2016) e os mesmos passaram a ser concebidos como uma arma intrínseca à guerra moderna.

Ao mesmo tempo em que os conflitos se tornavam mais complexos e desenvolvia-se a RMA, cresceu o entendimento acerca de uma responsabilidade coletiva internacional para proteger civis e prestar assistência humanitária. Nesse sentido, as operações de paz, a partir de 1999, passaram a ser autorizadas a usar a força para proteger civis como um dos passos para a estabilização dos conflitos. A atuação das forças de paz em conflitos assimétricos e complexos impôs a necessidade de maior conhecimento da situação para o sucesso do planejamento e da execução de ações em cumprimento dos mandatos. A prioridade na proteção de civis e a impossibilidade de garanti-la em toda a área de operações também remeteu à necessidade de aprimorar a coleta e análise de informações para auxiliar operações de prevenção, preempção e/ou resposta a ações que ameaçassem fisicamente civis, peacekeepers, membros das agências das Nações Unidas, suas instalações e/ou agentes humanitários. No entanto, a ONU só passou a dotar as operações no terreno de maior capacidade tecnológica, incluindo os VANTs, na presente década.

Em 2000, o Relatório Brahimi destacou apenas a tecnologia da informação como facilitadora chave de vários objetivos das operações de paz apresentados e recomendou a implementação de uma estratégia comum pelos departamentos envolvidos com essas operações, incluindo o uso de sistemas de informação geográfica (UN 2000).

A partir de 2006, quando a Força da União Europeia (EUFOR) operou VANTs B-Hunter belgas durante o período eleitoral na RDC, a ONU iniciou tentativas para utilização desses equipamentos, o que só ocorreu em 2013 (UN 2014b).

A doutrina de operações de paz, chamada 'Capstone', não se dedicou ao tema, apenas indicando a responsabilidade do Department of Field Support (DFS) 10 em prover o apoio em pessoal, finanças, aquisições, logística, comunicações e tecnologia da informação para as operações de paz (UN 2008).

9 Significa "o processo de seleção e priorização de alvos e correspondência com a resposta adequada, considerando os requisitos e capacidades operacionais” (Office of the Chairman of the Joint Chiefs of Staff 2020, 211, tradução nossa). "The process of selecting and prioritizing targets and matching the appropriate response to them, considering operational requirements and capabilities.”

10 Renomeado Department of Operational Support (DOS) em 2018. 
Em 2013, o Conselho de Segurança (CSNU) autorizou o uso de VANTs pela MONUSCO (UN 2013a). No ano seguinte, o Comitê Especial sobre Operações de Paz (C34) reconheceu o uso de tecnologia moderna para melhorar a consciência situacional e aumentar a segurança e proteção das forças de paz e solicitou que o Secretariado tomasse medidas necessárias para assegurar que as tropas e unidades no terreno fossem capazes de, efetivamente, responder aos desafios de segurança, incluindo o uso de equipamentos de proteção e de alta tecnologia ${ }^{11}$ (UN 2014b).

Em consequência, o Department of Peacekeeping Operations (DPKO) ${ }^{12}$ e o DFS estabeleceram um grupo de trabalho para considerar novas tecnologias e inovações e desenvolver uma estrutura de políticas em relação ao tema, de modo a assegurar parâmetros claros para a coleta, armazenamento seguro e manuseio adequado dos dados, além do respeito à confidencialidade (UN 2015a).

O Secretário Geral das Nações Unidas (SGNU) nomeou um Painel de Especialistas sobre Tecnologia e Inovação que, em seu relatório, admitiu que as forças de paz se mostravam incapazes de cumprir determinadas tarefas impostas pelo CSNU, em muitos casos, por não ter ferramentas adequadas como equipamentos de vigilância noturna, capacidade tecnológica para monitoramento remoto, além de pouca ou nenhuma infraestrutura para vigilância aérea não tripulada e sensores de solo não supervisionados. Concluindo que os VANTs podem trazer vantagens decisivas para uma operação nas áreas de segurança, segurança situacional e comando e controle, o Painel recomendou investimentos para equipar as missões com tecnologias atualizadas de comunicações e pequenos veículos aéreos não tripulados desarmados (UN 2014b).

O relatório do Painel de Independente de Alto Nível sobre Operações de Paz indicou atenção especial para capacidades, tecnologias e preparação de força necessárias para atender ambientes de ameaças assimétricas e a necessidade das missões usarem tecnologia moderna. Com relação à tecnologia e inovação, o Painel reforçou a introdução de tecnologias disponíveis e acessíveis para aumentar a eficiência e a eficácia das estruturas e serviços básicos para o sucesso das missões. O Painel salientou que as tecnologias não deveriam ser um fim em si mesmas, mas sim focadas no usuário, confiáveis em relação às condições no terreno, e recomendou priorização no seu fornecimento para as operações (UN 2015b).

11 O Comitê salientou que a utilização da tecnologia deveria respeitar os princípios consagrados na Carta da ONU sobre soberania e integridade territorial dos Estados-membros e o consentimento do Estado anfitrião.

12 Renomeado Department of Peace Operation (DPO) em 2018. 
O relatório Improving Security of United Nations Peacekeepers, conhecido como 'Relatório Cruz', encomendado para analisar as causas de fatalidades nas operações de paz devido a atos violentos, e entregue em dezembro de 2017, recomendou o uso de pequenos drones para mitigar emboscadas, assim como reunir informações antecipadas à frente dos comboios como forma de mitigar as ameaças por dispositivos explosivos improvisados. O relatório salientou que o uso efetivo de VANTs exige que habilidades aprofundadas como coleta e análise de informações sejam integradas às missões como um todo e que sensores de coleta de informações de alta tecnologia devem ser analisados em relação a sua adequabilidade aos ambientes operacionais. Por exemplo, em alguns cenários, VANTs táticos menores seriam mais úteis do que os sistemas grandes de VANTs. Ao mesmo tempo, o relatório indicou a necessidade das missões adquirirem outros equipamentos básicos para inteligência, como câmeras de alta qualidade (Cruz, Phillips e Cusimano 2017).

\section{O conflito e o emprego de VANTs na MINUSMA}

O Mali se tornou independente apenas em 1960. Dois anos após, ocorreu a primeira revolta tuaregue, grupo nômade que compõe cerca de $1 \%$ da população e vive principalmente no norte do país. Esse grupo protagonizou outras rebeliões na década de 1990, lideradas pela Frente Popular para a Libertação de Azawad (FPLA), e em 2006, quando vários grupos foram criados, incluindo o Movimento Nacional pela Libertação do Azawad (MNLA), que conseguiu, em um primeiro momento, reunir todas as facções rebeldes. Em janeiro de 2012, iniciou-se a quarta rebelião tuaregue, quando os rebeldes mostraram-se mais bem preparados, organizados e equipados (Benjaminsen 2008; Chauzal e Van Damme 2015). Além disso, novos grupos mostraram-se presentes. Militantes do MNLA, do grupo islâmico Ansar Dine ${ }^{13}$ e da organização terrorista Al Qaeda do Magrebe Islâmico (AQIM) ${ }^{14}$ realizaram vários ataques a instalações militares localizadas no norte do Mali e derrotaram as

13 Em 2011, Iyad ag Ghali foi negado como líder do MNLA devido, em parte, a sua radicalização e ao seu papel nos acordos firmados durante a terceira rebelião tuaregue. Diante disso, o ativista tuaregue criou o grupo islâmico Ansar Dine - 'defensores da fé' - alguns dias depois da primeira investida militar do MNLA, em janeiro de 2012. O Ansar Dine tem como objetivo implementar a sharia em todo o Mali (Chauzal e Van Damme 2015).

14 A AQIM foi criada em 1998, na Argélia, com o nome de Grupo Salafista de Pregação e Combate (Groupe Salafiste pour la Prédication et le Combat - GSPC), modificado em 2007 devido sua fusão com a Al Qaeda ocorrida no ano anterior. O grupo é ativo no Norte da África e na região do Sahel (Chauzal e Van Damme 2015). 
tropas malianas (Bleck e Michelitch 2015). No início de abril, o MNLA proclamou a independência da Azawad. ${ }^{15}$ No final daquele mês, todas cidades no norte do país já estavam tomadas. Entretanto, o MNLA começou a perder força e o Ansar Dine, apoiado pela AQIM e pelo Movimento pela Unidade e a Jihad na África Ocidental (MUJAO) ${ }^{16}$, ganhou força e passou a ver o MNLA como inimigo (Chauzal e Van Damme 2015). Nesse contexto, em 21 de março, militares malianos deram um golpe de Estado, mas a pressão internacional levou à constituição de um governo transitório (Bleck e Michelitch 2015; Cold-Ravnkilde 2013).

A situação caótica do país, o fortalecimento dos grupos islâmicos extremistas e a possibilidade deles tomarem a capital, Bamako, levaram o CSNU a autorizar o desdobramento da African-led International Support Mission to Mali (AFISMA), pela Comunidade Econômica dos Estados da África Ocidental (CEDEAO) e a União Africana (UA), a partir de setembro de 2013 (UN 2012).

Entretanto, em janeiro de 2013, os três grupos que tomaram a frente da rebelião (Ansar Dine, AQIM e MUJAO) avançaram para o sul do país e forçaram a retirada do exército maliano. A França lançou a 'Operação Serval', com cerca de 4.000 militares e, em 17 de janeiro, a CEDEAO apressou a instalação da AFISMA (Minusma 2018; Francis 2013). Com a derrota militar, os insurgentes dispersaram-se e recuaram para as montanhas e áreas desérticas, onde se reagruparam, reorganizaram-se e passaram a atacar tropas francesas e africanas presentes no país (Minusma 2018; Francis 2013). A França substituiu, então, a Operação Serval pela Operação Barkhane, desdobrada em conjunto com Burkina Faso, Chade, Mali, Mauritânia e Níger, que formam o G5 do Sahel, com o objetivo de regionalizar os esforços do combate ao terrorismo na região do Sahel (Sandvik 2015). A situação de instabilidade e insegurança culminou na criação da MINUSMA, em 25 de abril de 2013, pelo CSNU, a qual assumiu o controle da AFISMA e dos seus 6 mil militares a partir de $1^{\circ}$ de julho daquele ano (Minusma 2018; Boutellis 2015). De acordo com seu mandato, a MINUSMA deveria auxiliar na estabilização de áreas populacionais estratégicas, em especial a região norte do país, e apoiar a instituição da autoridade do Estado sobre o território maliano, tendo sido autorizada a usar a força para proteger civis e o pessoal da ONU e para garantir o cumprimento do mandato (UN 2013c).

15 O desejo dos Tuaregues, tanto do Mali quanto dos países vizinhos, pela implantação de um Estado independente - Azawad, abrangendo territórios por eles ocupados no norte do Mali, norte do Níger e sul da Argélia data da década de 1950 (Benjaminsen 2008).

16 O MUJAO surgiu em 2011, após uma cisão dentro da AQIM (Chauzal e Van Damme 2015). 
No entanto, a MINUSMA enfrentava problemas em avançar o processo político, os movimentos armados resistiam ao acantonamento de suas tropas e o governo se recusava a dar continuidade a medidas de construção de confiança (Boutellis 2015). Os ataques dos grupos islâmicos contra trabalhadores humanitários estrangeiros, membros da ONU e civis aumentaram (Nkala 2014) e a Missão tornou-se aquela com maior número de baixas de peacekeepers entre as que estavam em andamento (Cruz, Phillips e Cusimano 2017). Foi nesse quadro que a ONU autorizou a utilização de VANTs em apoio à operação de paz no Mali. A empresa contratada seria responsável por providenciar o pessoal para operar os equipamentos, garantindo suas próprias necessidades técnicas e de subsistência, e "operará em estreita colaboração com o pessoal da aviação e militar da ONU e interagir com o país anfitrião como e quando necessário"17 (UN 2014c, tradução nossa).

Em dezembro de 2015, a ONU firmou o contrato com a empresa inglesa Thales UK para o fornecer à MINUSMA três Hermes 900 - um modelo de VANT do tipo 'altitude média e longa duração' - 'medium-altitude long-endurance (MALE)'18, construído pela empresa israelense Israel's Elbit, duas estações de controle baseadas no aeroporto de Timbuktu e tripulações de voo, com o objetivo de usar simultaneamente duas aeronaves, mantendo uma em reserva (UN Flying Hermes 2016). Os voos operacionais começaram em julho de 2016 (De Charisey 2017).

De acordo com o contrato de aquisição, os VANTs não realizavam tarefas para operações militares. Assim, os dados coletados por eles não eram processados pela All Source Information Fusion Unit (ASIFU), unidade responsável pelo controle dos recursos operacionais de inteligência, vigilância e reconhecimento (intelligence, surveillance, and reconnaissance - ISR) e pela produção de produtos de inteligência solicitados pela liderança da MINUSMA com o objetivo de auxiliar o processo de tomada de decisão. Posteriormente, a ASIFU foi fundida com a Seção de Inteligência do Quartel General da Força Militar — Force Headquarters U2 (FHQ U2) ${ }^{19}$, sendo criada a Unidade Interina de Inteligência (IIU). Em 2017, a IIU passou por uma transição para começar a empregar um sistema personalizado desenvolvido pela Thales UK para a ONU (De Charisey 2017).

\footnotetext{
17 " [...] will operate closely with UN aviation and military personnel and interact with the host nation as and when required."

18 A categoria MALE classifica VANTs que voam a uma altitude de 10.000 a 30.000 pés por períodos entre 24 a 48 horas.

19 U2 é a seção do FHQ que lida com questões relativas à inteligência militar.
} 
Antes do desdobramento dos VANTs Hermes 900 sob controle da MINUSMA, algumas unidades militares utilizaram essa tecnologia em apoio às suas operações. As forças alemãs, por exemplo, desdobraram o modelo LUNA Tactical Unmanned Aircraft System (TUAS), da companhia alemã EMT Penzberg, e os modelos israelenses Heron 1s (De Charisey 2017). O modelo LUNA é um drone mais simples, podendo voar de 6 a 8 horas, enquanto o Heron 1s faz parte da categoria MALE e pode voar mais de 45 horas (Heron 2017; EMT 2009).

As tropas francesas da Operação Serval também já vinham utilizando VANTs do modelo de fabricação francesa Harfang, do tipo MALE e capacidade de sobrevoo de 24 horas. A Força Aérea francesa vinha utilizando o Harfang em várias de suas operações militares desde 2008, no Afeganistão e na Líbia. Ao detectar algumas desvantagens técnicas do modelo, no fim de 2013, a Força Aérea francesa adquiriu VANTs MQ-9 Reaper ${ }^{20}$, fabricados pela empresa estadunidense General Atomics, com capacidade de sobrevoo de 27 horas (Harfang MALE 2018; EADS Harfang 2018; Ahronheim 2016; France to Deploy 2013; General Atomics 2015). Ambos os VANTs foram utilizados pelas tropas francesas em missões de vigilância e reconhecimento, sendo que o Reaper foi utilizado também para targeting. Atualmente, a Operação Barkhane faz uso frequente de dois drones Reaper armados (Larrauri e Meier 2015). Outros tipos de VANTs de menor porte também foram utilizados no âmbito da MINUSMA. No Quadro 1 compilamos todos os VANTs que operam no Mali.

\section{Quadro 1. VANTS que operam no Mali}

\begin{tabular}{|c|c|c|c|}
\hline FROTA & TIPO & EMPRESA & NACIONALIDADE \\
\hline 3 & Hermes 900 & Thales UK & Reino Unido \\
3 & Heron 1s & Israel Aerospace Industries (IAI) & Israel \\
2 & MQ-9 Reaper & General Atomics & Estado Unidos \\
10 & Luna & EMT Penzberg & Alemanha \\
4 & Aladin & EMT Penzberg & Alemanha \\
9 & Mikado (AirRobot AR 100-B) & EMT Penzberg & Alemanha \\
3 & RQ-7 Shadow & AAI Corporation & Estado Unidos \\
4 & RQ-20 Puma & AeroVironment & Estado Unidos \\
4 & RQ-12A Wasp & AeroVironment & Estado Unidos \\
6 & RQ-11B Raven & AeroVironment & Estado Unidos \\
\hline
\end{tabular}

Elaboração própria com base em: Ahmed (2017), UN Flying Hermes (2016), Larrauri e Meier (2015) e De Charisey (2017).

20 Também chamado de Predator B, em referência ao famoso predador estadunidense. 


\section{Discussões sobre o emprego de VANTS}

Como dito anteriormente, selecionamos os elementos doutrina, organização, custos, domínio da informação, comando e controle, simulação e treinamento, e agilidade/ímpeto para discutir o emprego de VANTs na MINUSMA e nas operações de paz. Em termos militares, a doutrina visa combinar meios, capacidades e tecnologias de maneira que maximize vantagens. As guerras de guerrilha resultaram em doutrinas para operações de contra insurreição. Há casos de avanços tecnológicos que resultaram em avanços doutrinais, como o advento dos aviões e das armas nucleares. No caso da ONU, após décadas de desdobramentos de operações de paz foi firmada uma doutrina (Capstone) a qual não abarcou o uso de VANTs. O contexto de algumas operações de paz (RDC e Mali) levaram à utilização da tecnologia e as lições apreendidas resultaram na publicação das primeiras diretrizes para a geração e o emprego dessas capacidades somente em fevereiro de 2019.

O documento United Nations Use of Unmanned Aircraft Systems (UAS) Capabilities tratou de 'sistemas de aeronaves não tripuladas', ou seja, todos os componentes que incluem uma ou mais aeronaves não tripuladas, a rede de apoio e o equipamento e pessoal necessários para controlar essas aeronaves. Os sistemas disponíveis foram categorizados de acordo com o Manual de Aviação da ONU e com o contexto militar. O documento apresentou considerações gerais sobre geração e desdobramento desses sistemas e deixou claro que a decisão de seu emprego deve basear-se nos impactos operacionais, técnicos, ambientais, financeiros e políticos dos VANTs nas operações de paz, bem como a necessidade das diretrizes serem observadas em conjunto com os documentos da ONU que tratam de inteligência e aviação (UN 2019).

A doutrina de operações de paz não foi modificada por conta da utilização dos VANTs. A MINUSMA combina os meios e capacidades em seus planejamentos e de acordo com suas prioridades estratégicas e as dinâmicas do conflito.

A estrutura das organizações deve evoluir de forma a apoiar as tecnologias e doutrinas. Na ONU, mudanças estruturais são normalmente lentas em razão do processo decisório que envolve tanto os diversos departamentos como (e principalmente) os Estados-membros. Após cinco anos de utilização de VANTs, foi estabelecida uma Célula Conjunta com experts do DPO e do DOS para apoiar a geração e emprego dessa tecnologia nas operações de paz. A partir das diretrizes sobre sistemas de VANTs, as questões organizacionais deverão aparecer mais 
claramente na estrutura das operações de campo. No caso da MINUSMA, os VANTs foram desdobrados em conjunção com a estrutura de inteligência, até então inédita nas operações de paz. Dessa forma, a ASIFU ficou diretamente ligada ao Comandante da Força Militar21, que controlou o uso dos VANTs. Dessa forma, eles resultaram em pequenas adaptações na estrutura da operação.

A criação, manutenção e mudanças nas operações de paz sofrem imposições orçamentárias. Para o emprego de VANTs na MINUSMA, o contrato firmado com a empresa privada foi no valor de 24,2 milhões de dólares para três anos de fornecimento dos equipamentos, operadores e bases de operação (Dombe 2016). Isso significou pouco mais de oito milhões/ano, representando 1,31\% do orçamento anual da Missão, que era de 1,048 bilhão de dólares. Assim, pode-se dizer que o custo da implantação de VANTs foi modesto, o que tende a estimular seu uso. Mesmo que o aumento de VANTs ou de bases para suprir a necessidade de cobertura de uma área maior no Mali significasse aumento nos custos da missão de paz, o que é sempre um desafio, ainda assim seria mais barato que utilizar outros ativos como helicópteros para cumprir tarefas semelhantes.

Em razão das características do conflito no Mali, a MINUSMA teve a participação de vários contingentes europeus que utilizaram VANTs próprios. Discutiu-se a inclusão de VANTs nos requerimentos para os países contribuintes (Troop Contributing Countries - TCCs) como uma política sistemática do DPO, ou seja, que determinados contingentes fossem desdobrados com essa tecnologia. No entanto, isso resultaria em dependência da ONU para com os países que detêm esse tipo de tecnologia e que se dispusessem a fornecê-los, o que restringiria a participação dos que não a possuem. ${ }^{22}$

Com relação ao terceiro elemento - o domínio da informação - são discutidos dois pontos: o conhecimento situacional e o uso das informações coletadas. A complexidade dos ambientes em que as missões de paz atuais estão desdobradas e a amplitude das tarefas que devem cumprir tornaram imperativo o melhor conhecimento possível sobre a situação no terreno por parte dos peacekeepers e dos tomadores de decisão. A melhor consciência situacional resulta em decisões mais meticulosas sobre onde e como desdobrar as tropas, proporcionando-lhes

21 A ASIFU tinha duas companhias de ISR desdobradas no Setor Leste - Gao, da Holanda, e no Setor Oeste Timbuktu, da Suécia.

22 Entrevista com ex-oficial sênior da ONU conduzida pelo autor em fevereiro de 2018, em Brasília-DF, sob condição de anonimato. 
maior segurança (Larrauri e Meier 2015). Isso ganha relevância se observamos que, das 3.767 vítimas fatais registradas em missões de paz desde 1948, 26,5\% foram causados por 'atos maliciosos'23. No caso da MINUSMA, apesar dos VANTs terem melhorado a capacidade dos peacekeepers conhecerem a situação no terreno, não há evidências sobre a diminuição das mortes por atos de violência devido ao seu uso.

Gráfico 1. Óbitos causados por Malicious Acts na MINUSMA

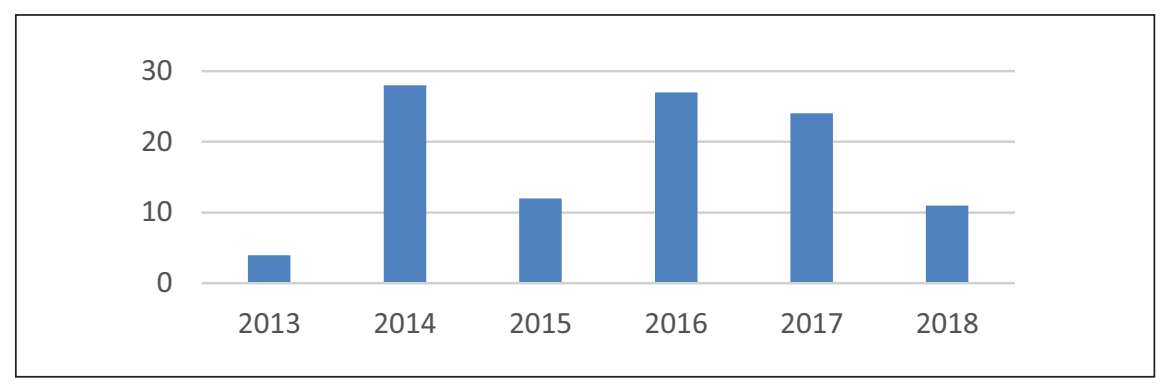

Fonte: UN (2018a)

Como pode-se verificar no gráfico, o número de óbitos na MINUSMA atingiu seu ápice em 2014. Em 2015, houve diminuição seguida de aumento brusco em 2016. O número ainda se manteve alto no ano seguinte quando os VANTs já estavam em operação na missão e, em 2018, ocorreu novamente uma queda.

Andrews (2017), salientou que os VANTs podem servir como mecanismo para a supervisão das próprias missões, no sentido de registrarem as ações de seus componentes. Além disso, o autor ponderou que a maior capacidade das missões de saber o que acontece no terreno, acarretaria mais responsabilidade dos comandantes no caso de ocorrerem emboscadas, massacres ou falta de alerta antecipado. As falhas ocasionariam escândalos que enfraqueceriam a confiança da população e do país anfitrião.

Se os peacekeepers tiverem aviso prévio ou razões para acreditarem que os movimentos rebeldes ameaçam civis e falharem em fazer "o suficiente" como julgado pela sociedade, a harmonia da Missão poderia ser irreparavelmente danificada. Isto adiciona elementos de risco à reputação e devem ser

23 Maliciou acts, em inglês. Terminologia usada nas Nações Unidas. O Relatório Cruz utilizou a expressão 'atos de violência'. Ambos os termos se referem a "atos de ameaça à segurança que resultam de conflitos armados, terrorismo, agitação civil violenta e crime” (Cruz, Phillips e Cusimano 2017, 2, tradução nossa; UN 2018b). "Acts of violence are the consequence of security threats that stem from armed conflict, terrorism, violent civil unrest and crime". 
considerados quando se usam UAVs em conflitos particularmente hostis e dinâmicos (Andrews 2017, 7, tradução nossa). ${ }^{24}$

Os VANTs agem como fator de dissuasão. Combatentes diminuiriam atos de violência por conta da possibilidade de serem registrados, de seus perpetradores serem identificados e sofrerem consequências legais ou ações das forças de paz como resposta (Pilgrim 2015). No entanto, a presença de VANTs poderia indicar a pouca probabilidade de ação dos peacekeepers naquele momento, incentivando atos violentos. Além disso, a operação de drones sem uma reação das tropas da missão, com o tempo, levaria à perda da credibilidade. Ou seja, a dissuasão só funcionaria se a identificação de VANTs por perpetradores de violência fosse "quase sempre e imediatamente seguida de alguma demonstração de força direta ou indireta”25. (Larrauri e Meier 2015, 43, tradução nossa). O território do Mali é bastante adverso e o conflito se concentrava na região norte do país, tornando a vigilância mais complexa. A ONU apresentou que os VANTs tipo MALE permitiram o monitoramento de áreas remotas e movimentos de grupos armados, dissuadindo a violência (UN 2015c).

A efetividade do uso dos VANTs está relacionada com a capacidade da operação de paz analisar, de maneira eficiente, os dados coletados para auxiliar o planejamento das ações que serão tomadas. A ONU utiliza três canais de coleta de informação: a inteligência de código aberto (UN open-source intelligence UN-OSINT); a inteligência humana (UN human intelligence - UN-HUMINT), que pode ser obtida por meio do contato com a população; e a inteligência de imagens (UN imagery intelligence - UN-IMINT), que inclui os dados coletados pelos VANTs (Abilova e Novosseloff 2016).

Abilova e Novosseloff (2016) abordaram o problema da ONU quanto à coleta de um enorme número de informações sem a concomitante capacidade de análise destas:

Ferramentas tecnológicas não fornecem respostas ou informações automáticas; seu uso adequado requer perícia. Uma coisa é obter dados brutos e outra, bem diferente, é analisá-los adequadamente em busca de um objetivo específico. Até agora, a ONU tendeu a concentrar-se excessivamente na coleta de informações sem dar atenção suficiente ao processamento,

24 "If peacekeepers have advanced warning or reason to believe rebel movements threaten civilians and fail to do 'enough' as judged by society, mission rapport could be irreparably damaged. This adds elements of reputational risk and should be considered when using UAVs in particularly hostile and dynamic conflicts."

25 "[...] almost always and immediately followed by some direct or indirect show of force." 
exploração, análise e compartilhamento. O Painel de Especialistas em Tecnologia e Inovação da ONU em Manutenção da Paz observou que as missões frequentemente se encontram presas no "fog of more" 26 , por meio do qual uma sobrecarga de informações aumenta a incerteza sobre o que priorizar (Abilova e Novosseloff 2016, 13, tradução nossa). ${ }^{27}$

As autoras apresentaram uma questão que é recorrente nas missões de paz multidimensionais, que os Joint Mission Analysis Centre (JMAC) têm acesso às informações que precisam, "mas não têm capacidade para analisá-las e transformá-las em planos"28. Nesse sentido, elas afirmaram que "melhorar a capacidade analítica das missões provou ser mais difícil do que melhorar sua capacidade tecnológica”29 e que "o maior uso de tecnologia para coletar informações, portanto, vem com maior necessidade de recursos analíticos para transformar essas informações em inteligência”30 (Abilova; Novosseloff 2016, 13, tradução nossa).

Na MINUSMA, a unidade de Inteligência do contingente sueco possuía cerca de 250 membros. Segundo ex-oficial sênior da ONU, “o produto do trabalho desses funcionários, que era de primeira linha, não era compartilhado corretamente com outras seções ou unidades da MINUSMA”. Em consequência, “o excelente trabalho não era aproveitado em prol da missão como um todo”. ${ }^{31} \mathrm{O}$ JMAC trabalhava para o Chefe da Missão e utilizava dados obtidos pelos VANTs em suas análises. No entanto, foi questionada a efetiva contribuição dessas análises para as ações das tropas desdobradas no terreno. ${ }^{32}$

Segundo o SGNU, a MINUSMA adotou uma série de medidas para otimizar suas atividades de inteligência em 2018:

26 Em tradução livre: 'nevoeiro do excesso'.

27 "Technological tools do not provide automatic answers or information; their proper use requires expertise. It is one thing to get raw data and quite another to properly analyze it in pursuit of a specific objective. So far, the UN has tended to over-focus on collecting information without giving sufficient attention to processing, exploiting, analyzing, and sharing it. The UN Expert Panel on Technology and Innovation in UN Peacekeeping noted that missions often find themselves caught up in the "fog of more," whereby an overload of information increases uncertainty about what to prioritize."

28 "[...] but lack the capacity to analyze it and transform it into plans."

29 "Improving the analytical capacity of missions has proven more difficult than improving their technological capacity."

30 "Greater use of technology to collect information therefore comes with a greater need for analytical capabilities to transform this information into intelligence."

31 Entrevista com ex-oficial sênior da ONU conduzida pelo autor em fevereiro de 2018, em Adis Ababa — Etiópia, sob condição de anonimato.

32 Entrevista com ex-oficial da MINUSMA conduzida pelo autor em novembro de 2018, no Rio de Janeiro - RJ, sob condição de anonimato. 
Estabeleceu uma rede de comunicação segura para apoiar as capacidades relacionadas à inteligência da Missão em três locais, com expansão planejada para duas sedes setoriais adicionais. Veículos aéreos não tripulados de alta e média altitude estavam em operação em Timbuktu, fornecendo capacidade de informação e análise. Como complemento do sistema de vigilância de campo em Kidal, um veículo aéreo não tripulado tático foi desdobrado para fornecer vigilância estendida além do perímetro do campo, complementando o sistema de vigilância de circuito fechado de televisão, atualmente estabelecido nas principais instalações das Nações Unidas. A infraestrutura de inteligência e informação da Missão foi projetada para aumentar a conscientização e a detecção antecipada de ameaças a civis e para garantir a proteção dos campos da MINUSMA e do pessoal associado (UN 2018c, 14, tradução nossa). ${ }^{33}$

Em meados daquele ano, o CSNU listou medidas que seriam tomadas em prol de maior proteção do pessoal da Missão, entre as quais estava o aprimoramento das capacidades de inteligência e análise da MINUSMA, incluindo as capacidades de vigilância e monitoramento por meio do "uso de tecnologia moderna, como sensores múltiplos, fusão de inteligência e sistemas aéreos não tripulados, bem como a exploração de rotas alternativas de suprimento logístico” (UN 2018d, 15, tradução nossa). ${ }^{34}$

A questão sobre o uso dos dados coletados é sem dúvida delicada. Tanto o Painel de Especialistas como o C34 apresentaram a preocupação de que o uso de VANTs não pode comprometer a soberania política e territorial dos Estados. A preocupação dos governos dos países anfitriões e seus vizinhos sobre o desdobramento dessa tecnologia leva a um processo permeado por muita negociação. Outra questão se refere à propriedade dos dados coletados. Dados e imagens coletados pelos VANTs "se tornam propriedade da ONU e estão sujeitos aos mesmos direitos, proteções e regimes de confidencialidade que outras propriedades da ONU”35

33 "MINUSMA established a secure communication network to support the Mission's intelligence-related capacities in three locations with a planned expansion to two additional sector headquarters. Medium-altitude long-endurance unmanned aerial vehicles were in operation in Timbuktu, providing information and analysis capability. As a complement to the camp surveillance system in Kidal, one tactical unmanned aerial vehicle was deployed to provide extended surveillance beyond the camp perimeter, complementing the closed-circuit television surveillance system currently being established in the main United Nations premises. The Mission's intelligence and information infrastructure is designed to increase awareness of and the early detection of threats to civilians and to ensure the protection of MINUSMA camps and associated personnel."

34 "[...] use of modern technology such as multiple sensors, intelligence fusion and unmanned aerial systems, as well as by exploring potential alternative logistical supply routes."

35 "[...] becomes UN property and is subject to the same rights, protections and confidentiality regimes as other UN property.” 
(Larrauri e Meier 2015, 51, tradução nossa). Adiciona-se às preocupações, a possibilidade de vazamento de informações, o que poderia gerar alto grau de desconfiança quanto à eficiência da ONU em manter a confidencialidade dos dados (Charbonneau 2015). Mesmo que durante as operações a ONU mantenha a confidencialidade, ela não estaria garantida após o encerramento da missão. Na MINUSMA, tanto o compartilhamento de dados como o acesso a bases de dados, incluindo aqueles obtidos pelos VANTs, foi comprometido. Alguns contingentes, inclusive, apresentavam restrições formais com relação a essas questões como forma de preservar a confidencialidade de equipamentos e procedimentos (ver Rietjens e de Waard 2017).

Com relação ao comando e controle dos VANTs, as Diretrizes do DPO/DOS afirmaram que as operações deles "são mais eficazes quando o controle da capacidade é delegado ao nível mais baixo possível”, mas que a natureza das operações é que vai ditar como isso se dará e os tipos de VANTs a serem utilizados (UN 2019, 11, tradução nossa). ${ }^{36}$ No caso da MINUSMA, as unidades que possuíam VANTs mantiveram o comando e controle sobre eles. Os VANTs contratados pela ONU foram utilizados pelo setor de inteligência, que abastecia o Comandante da Força (níveis operacional e tático) e, a partir do componente militar, abastecia o Chefe da Missão, por meio do JMAC (níveis operacional e estratégico).

Através da simulação e treinamento é que se combinam as habilidades humanas com capacidades tecnológicas. As Diretrizes da ONU sobre VANTs salientaram que, além da capacidade de operar esses equipamentos, deve haver analistas altamente especializados em imagens. Assim, os provedores de VANTs para as operações de paz devem incluir treinamento e educação dentre os componentes mínimos do serviço a ser prestado (UN 2019). Na MINUSMA, os operadores e analistas eram experts em suas áreas, ou seja, foram desdobrados com conhecimento e treinamento prévio para o emprego da tecnologia, por conta do contrato firmado pela ONU.

A agilidade inclui capacidades, sistemas e tecnologias projetados para colocar uma força em campo e mantê-la lá, ou seja, envolve mobilidade e sustentação. Os VANTs podem tornar tarefas mais fáceis ou permitir que tarefas impostas pelo CSNU (mandatos) sejam melhor cumpridas. Por exemplo, aumentam a habilidade dos peacekeepers de monitorar violações dos direitos humanos e responder a elas e podem ajudar no desdobramento mais rápido de tropas para a proteção de

36 “[...] are most effective when the control of the capability is delegated to the lowest possible level". 
civis, a partir das imagens transmitidas (e suas análises). Ou seja, auxiliam na diminuição do tempo de resposta.

A MINUSMA recebeu a tarefa de proteger civis, promover e proteger direitos humanos e apoiar a assistência humanitária. Já em 2014, o SGNU atribuiu a melhora nas medidas adotadas para a proteção de civis, principalmente, aos multiplicadores de força, incluindo os VANTs que haviam iniciado voos de vigilância no período coberto pelo Relatório (UN 2014d). Em março de 2015, o SGNU apresentou uma relação entre a disputa pelo controle de rotas comerciais e de tráfico e os confrontos que ocorriam na região norte, a partir de imagens coletadas pelos VANTs (UN 2015d). O Relatório de 28 de março de 2016 informou terem sido realizadas 26 missões de escoltas com o apoio de ativos de aviação, VANTs e forças especiais, o que teria possibilitado “a construção dos campos da MINUSMA, a reabertura do aeródromo de Kidal e a manutenção da Missão naquelas regiões”37 (UN, 2016a, 13, tradução nossa).

No entanto, o SGNU admitiu que

Em Gao e Timbuktu, apenas 8 veículos aéreos não tripulados dos 38 desdobrados para a Missão poderiam ser usados para fornecer vigilância de curto e médio alcance, devido ao número limitado de estações de controle terrestre. Para resolver essas deficiências, a Missão adquiriu em junho um sistema aéreo não-tripulado com um alcance de $600 \mathrm{~km}$, que foi posteriormente desdobrado em Timbuktu. Outros dois, um dos quais seria usado como um complemento, entrariam em operação em outubro (UN, 2016b, 14, tradução nossa). ${ }^{38}$

A literatura sobre inovação militar aborda a questão do ímpeto para mudanças e que ele pode estar relacionado a feedbacks positivos ou negativos vindos do terreno (Rosen 1991). No caso da MINUSMA, pode-se arguir que o elevado número de baixas foi um fator que compeliu a ONU e seus Estados-membros a autorizar o uso da tecnologia. No mesmo sentido, o feedback positivo do uso dos VANTs no terreno facilitou a incorporação da tecnologia pela Organização em suas operações de paz.

37 " $[\ldots]$ the construction of MINUSMA camps, the reopening of the Kidal airfield and the sustainment of the Mission in the regions."

38 "In Gao and Timbuktu, only 8 unmanned aerial vehicles out of the 38 deployed to the Mission could be used to provide short- and medium-range surveillance, owing to the limited number of ground control stations. To address these shortcomings, the Mission acquired in June one unmanned aerial system with a range of $600 \mathrm{~km}$, which was subsequently deployed to Timbuktu. Another two, one of which would be used as a spare, would be operational by October." 
Conforme salientou o então Vice-Secretário Geral para Operações de Paz, Hervé Ladsous, a introdução de VANTs teria sido uma demonstração de que a ONU é capaz de se modernizar e usar recentes tecnologias para monitorar movimentos de grupos armados e permitir melhor proteção das populações vulneráveis. Indicou que a ação mais proativa das operações de paz exigiam capacidade de responder rapidamente, uma vez que não era possível cobrir grandes áreas geográficas e que maior mobilidade e agilidade exigiam novos tipos de capacidades, equipamentos e tecnologias. Então, os multiplicadores de forças, incluindo os VANTs, seriam "fundamentais para fornecer às missões a mobilidade, capacidade de reação rápida e robustez necessárias para implementar mandatos complexos”39 (Ladsous 2014, 9, tradução nossa).

\section{Conclusão}

As inovações na ONU são normalmente demoradas, o que é compreensível em razão de ser uma organização intergovernamental global e de que suas decisões necessitam ser discutidas e aprovadas pelos Estados-membros. Se a literatura sobre inovação militar apresenta dificuldades e desafios para forças armadas nacionais inovarem, no caso das operações de paz há outros complicadores como os interesses e restrições dos membros da ONU e o fato das operações funcionarem com tropas e policiais fornecidos por diferentes países com diferentes capacidades, mas que deveriam trabalhar para atingir um objetivo comum, ou seja, o cumprimento das tarefas requeridas pelos mandatos.

Num curso normal, a doutrina deveria informar as estruturas necessárias e os equipamentos a serem desenvolvidos. No entanto, foi a necessidade imposta pela complexidade no terreno que levou ao desdobramento dos VANTs na MINUSMA, sem que tenha ocorrido qualquer alteração doutrinária nas operações de paz. O uso dessa tecnologia pode ser entendido como um 'movimento adaptativo' que a ONU realizou para melhorar a posição da operação no Mali em relação aos beligerantes e, com isso, avançar no processo de paz. O desdobramento dos VANTs, em conjunto com componente robusto de inteligência, resultou na adequação da estrutura da Missão em nível operacional e tático.

39 "[...] central to providing missions with the necessary mobility, rapid reaction capacity, and robustness needed to implement complex mandates.” 
Os custos dos VANTs foram relativamente baixos se comparados ao orçamento total da MINUSMA. O domínio da informação continua sendo sensível. Se por um lado, os VANTs auxiliaram sobremaneira o conhecimento situacional, por outro lado, alguns equipamentos utilizados e os produtos gerados por eles, sendo considerados sigilosos, impactaram negativamente a sua utilização e o compartilhamento das informações por eles geradas. O comando e controle dos VANTs foi delegado ao nível mais baixo possível em decorrência das estruturas da MINUSMA. O treinamento e simulação não impactou o uso da tecnologia, uma vez que todos os envolvidos eram experts e com o necessário background. A agilidade/ímpeto para o uso da tecnologia pode ser vista por dois prismas. Em termos políticos, foi demorada e com pouco ímpeto por conta dos entendimentos que se fazem necessários para a implementação de qualquer mudança na sede da ONU em Nova Iorque. No entanto, a implementação no terreno foi ágil, principalmente por conta dos benefícios que os VANTs trariam para as operações no terreno.

O feedback positivo de seu emprego (juntamente com o caso da RDC) nutriram o DPO para preparar as diretrizes que passaram a nortear o processo de geração e desdobramento de VANTs, a partir de fevereiro de 2019.

A introdução de VANTs na MINUSMA não exigiu mudanças mais profundas, mas sim, aumentou a eficácia/eficiência da Missão em cumprir suas funções tradicionais. Dessa forma, tratou-se de uma inovação adaptativa. O caso da MINUSMA pode ser visto como facilitador da inovação no âmbito das operações de paz. Os ganhos operacionais e os desafios sobre o seu emprego na MINUSMA ajudaram no aperfeiçoamento normativo da ONU e, provavelmente, ajudarão nos futuros desdobramentos de VANTs em outras operações de paz.

\section{Referências}

Abilova, Olga e Novosseloff, Alexandra. 2016. "Demystifying Intelligence in UN Peace Operations: Toward an Organizational Doctrine”. New York: International Peace Institute. Disponível em: <https://ssrn.com/abstract=2893281 > . Acesso em: 28 jul. 2020.

Ahmed, Ovais. 2017. "Second Remotely Piloted Aircraft System (RPAS) Symposium". International Civil Aviation Organization (ICAO). Disponível em: < https://www. icao.int/Meetings/RPAS17/Presentations/1.3.3\%200vais\%20Ahmed.pdf > . Acesso em: 28 jul. 2020. 
Ahronheim, Anna. 2016. “Israel's Heron drone completes first successful mission in Mali”. The Jerusalem Post. Disponível em: < https://www.jpost.com/Israel-News/ Israels-Heron-drone-completes-first-successful-mission-in-Mali-471961 > . Acesso em: 28 jul. 2020.

Andrews, Sandra. M. 2017. "Drones in the DRC: A Case Study for Future Deployment in United Nations Peacekeeping”. Intersect 10, no. 2: 1-10.

Arreguín-Toft, Ivan. 2005. How the weak win wars: a theory of asymmetric conflict. New York Cambridge University Press.

Arthur, Brian W. 2009. The Nature of Technology: What it is and how it evolves. London: Penguin Books.

Benjaminsen, Tor A. 2008. "Does Supply-Induced Scarcity Drive Violent Conflicts in the African Sahel? The Case of the Tuareg Rebellion in Northern Mali”. Journal of Peace Research 45, no. 6: 819-836.

Bleck, Jamie e Michelitch, Kristin. 2015. "The 2012 Crisis in Mali: Ongoing Empirical State Failure”. African Affairs 114(457): 598-623.

Boutellis, Arthur. 2015. "Can the UN Stabilize Mali? Towards a UN Stabilization Doctrine?" Stability: International Journal of Security \& Development 4, no. 1: 1-16.

Chamayou, G. 2015. Teoria do drone. São Paulo: Cosac Naify.

Charbonneau, Louis. 2015. “U.N. panel urges increased use of drones in peacekeeping missions”. Reuters. Disponível em: < http://www.reuters.com/article/us-unpeacekeepers-drones-idUSKBN0LR24G20150223 > . Acesso em: 28 jul. 2020.

Chauzal, Grégory e Van Damme, Thibault. 2015. “The roots of Mali’s conflict: Moving beyond the 2012 crisis”. Netherlands Institute of International Relations Clingendael. Conflict Research Unit. Disponível em: < https://www.clingendael.org/pub/2015/ the_roots_of_malis_conflict/ > . Acesso em:28 jul. 2020.

Cohen, Eliot A. 2004. "Change and Transformation in Military Affairs". The Journal of Strategic Studies 27, no. 3:395-407.

Cold-Ravnkilde, Signe M. 2013. "War And peace in Mali: Background and perspectives”. Danish Institute for International Studies Report, Copenhagen. Disponível em: $<$ https://www.diis.dk/files/media/publications/import/extra/rp2013-33_mali-signemarie-cold-ravnkilde_web_1.pdf > . Acesso em: 28 jul. 2020.

Cole, Chris. "Rise of the Reapers: A brief history of drones. Drone Wars UK, United Kingdom”. Disponível em: < https://dronewars.net/2014/10/06/rise-of-the-reapersa-brief-history-of-drones/ > . Acesso em: 28 jul. 2020.

Cooke, Tim. 2017. A Timeline of Military Robots and Drones. North Mankato: Capstone Press.

Cruz, Carlos A. Santos, Phillips, William R. e Cusimano, Salvator. 2017. "Improving Security of United Nations Peacekeepers: we need to change the way we are doing business”. Independent Report. New York. 
De Charisey, E. 2017. Desert watchers: MINUSMA's intelligence capabilities. Jane's Defence Weekly 54, no. 23. Disponível em: < http://www.janes.com/images/assets/ 319/71319/Desert_watchers_MINUSMA_s_ intelligence_capabilities.pdf > . Acesso em: 10 out. 2018.

Deshpande, Vikrant. 2018. Hybrid Warfare: The Changing Character of Conflict. New Delhi: Institute for Defence Studies \& Analyses.

Diálogo Setorial União Europeia-Brasil. 2017. Estudo sobre a indústria brasileira e europeia de Veículos Aéreos Não-tripulados. Disponível em: < http://www.mdic.gov.br/images/ publicacao_DRONES-20161130-20012017-web.pdf > . Acesso em: 30 set. 2017.

Dombe, Ami Rojkes. 2016. “Hermes 900 to support UN Mission in Mali”. IsraelDefense. Disponível em: < https://www.israeldefense.co.il/en/content/hermes-900-supportun-mission-mali > . Acesso em: 01 mar. 2019.

Duarte, Érico. 2012. Conduta da Guerra na Era Digital e Suas Implicações para o Brasil: Uma Análise de Conceitos, Políticas e Práticas de Defesa. Brasília: Ipea.

EADS Harfang. 2018. “Military Factory”. Disponível em: < https://www.militaryfactory. com/aircraft/detail.asp?aircraft_id=1049\#specs > . Acesso em: 10 ago. 2018.

EMT. 2009. "LUNA Aerial Reconnaissance and Surveillance System”. Disponível em: < https://www.emt-penzberg.de/uploads/media/LUNA_en.pdf > . Acesso em: 28 jun. 2018.

Ernst, Dieter, Mytelka, Lynn K. e Ganiatsos, Tom. 1998. “Technological capabilities in the context of export-led growth: a conceptual framework”. In Ernst, Dieter, Ganiatsos, Tom e Mytelka, Lynn K. (eds.). Technological Capabilities and Export Success in Asia. London: Routledge.

Farrell, Theo, Rynning, Sten e Terriff, Terry. 2013. Transforming Military Power since the Cold War: Britain, France, and the United States, 1991-2012. Cambridge: Cambridge University Press.

“France to Deploy Two U.S.-made Reaper drones in the Sahel”. 2013. EU Bulletin. Disponível em: < https://www.eubulletin.com/1726-france-deploy-two-u-s-madereaper-drones-sahel.html > . Acesso em: 10 ago. 2018.

Francis, David J. 2013. The regional impact of the armed conflict and French intervention in Mali. Norwegian Peacebuilding Resource Centre, [s. I.]:1-16.

Fridman, Ofer. 2018. Russian "Hybrid Warfare": Resurgence and Politicization. Oxford: Oxford University Press.

General Atomics. 2015. "MQ-9 Reaper/Predator B”. Disponível em: < https://www.gaasi.com/predator-b > . Acesso em: 28 jul. 2020.

Gilman, Daniel. 2014. “Unmanned Aerial Vehicles in Humanitarian Response”. Nations Office for the Coordination of Humanitarian Affairs. Disponível em: < https:// www.unocha.org/fr/publication/policy-briefs-studies/unmanned-aerial-vehicleshumanitarian-response > . Acesso em: 28 jul. 2020. 
Grissom, Adam. 2006. The future of military innovation studies. Journal of Strategic Studies 29, no. 5: 905-934.

“Harfang MALE Unmanned Aerial Vehicle (UAV)”. Air Force Technology. 2018. Disponível em: < https://www.airforce-technology.com/projects/harfang-drone/\#: : text = Harfang \% 20is \% 20a \% 20medium \% 2Daltitude, the \% 20depth \% 20 of \% 20the \% 20 battlefield. > . Acesso em: 28 jul. 2020.

“Heron”. 2017. Israel Aerospace Industries. Disponível em: < https://www.iai.co.il/p/ heron > . Acesso em: 29 jul. 2020.

Ladsous, Hervé. 2014. New Challenges and Priorities for UN Peacekeeping. The Brookings Institution. Disponível em: < https://peacekeeping.un.org/sites/default/files/hlremarks-brookings.pdf > . Acesso em: 17 nov. 2018

Larrauri, Helena Puig e Meier, Patrick. 2015. "Peacekeepers in the Sky: The Use of Unmanned Unarmed Aerial Vehicles for Peacekeeping”. ICT4Peace Foundation. Disponível em: < https://ict4peace.org/wp-content/uploads/2015/09/Peacekeepersin-the-Sky1.pdf $>$. Acesso em: 17 jul. 2018.

Magella, Paulo Eduardo Albuquerque de. 2016. A operação de aeronaves remotamente pilotadas e a segurança do espaço aéreo. Rio de Janeiro: ESG.

Mazzar, Michael, Schaffer, Jeffrey e Ederington, Benjamin. 1993. Military Technical Revolution. Washington: Center for Strategic and International Studies.

MINUSMA. 2018. "History”. UN. Disponível em: < https://minusma.unmissions.org/ en/history > . Acesso em: 28 jul. 2020.

Mytelka, Lynn K. 2000. Local Systems of Innovation in a Globalized World Economy. Industry and Innovation 7, no. 1: 15-32.

Nkala, Oscar. 2014. “UN to deploy unarmed surveillance drones in Mali”. Defence Web. Disponível em: < https://www.defenceweb.co.za/aerospace/aerospace-aerospace/ un-to-deploy-unarmed-surveillance-drones-in-mali/ > . Acesso em: 28 jul. 2020.

Nonami, Kenzo, Kendoul, Farid, Suzuki, Satoshi, Wang, Wei e Nakazawa, Daisuke. 2010 Autonomous Flying Robots: Unmanned Aerial Vehicles and Micro Aerial Vehicles. Tokyo: Springer.

Office of the Chairman of the Joint Chiefs of Staff. 2020. DOD Dictionary of Military and Associated Terms. Disponível em: < https://www.jcs.mil/Portals/36/ Documents/ Doctrine/pubs/dictionary.pdf > . Acesso em: 28 jul. 2020.

Oliveira, Francisco M. E. 2016. Unmanned Aerial Systems (UAS): Questões Legais e Éticas da sua utilização no combate ao terrorismo. Dissertação: Mestrado em Direito e Segurança. Faculdade de Direito. Lisboa: Universidade Nova de Lisboa.

Peron, Alcides Eduardo dos Reis. 2016. American way of war: o reordenamento sociotécnico dos conflitos contemporâneos e o uso de drones. Tese (doutorado). Instituto de Geociências. Campinas: UNICAMP. 
Pilgrim, Sophie. 2015. “Are UN drones the future of peacekeeping?” France 24. Disponível em: < https://www.france24.com/en/20150409-un-drones-future-peacekeepingdemocratic-republic-congo-fdlr-humanitarian-drc > . Acesso em: 02 out. 2018.

Rietjens, Sebastiaan e de Waard, Erik. 2017. “UN Peacekeeping Intelligence: The ASIFU Experiment”. International Journal of Intelligence and CounterIntelligence 30, no. 3: 532-556.

Rosen, Stephen P. 1991. Winning the Next War: Innovation and the Modern Military. Ithaca: Cornell University Press.

Rutherford, F. James e Ahlgren, Andrew. 1991. Science for All Americans. New York: Oxford University Press.

Sandvik, Kristin B. e Lohne, Kjersti. 2014. "The Rise of the Humanitarian Drone: Giving Content to an Emerging Concept”. Millennium: Journal of International Studies 43, no. 1: 145-164.

Schaub Jr, Gary. 2015. "JUSTAS for all? Innovation and UAVs in the Canadian Forces". Defence Studies 15, no. 2:124-142.

“UN Flying Hermes 900 UAVs in Mali”. 2016. Defence Web. Disponível em: < http://www. defenceweb.co.za/index.php?option $=$ com_content $\&$ view $=$ article\&id $=44843$ : unflying-hermes-900-uavs-in-mali\&catid $=35$ :Aerospace \&Itemid $=107>$. Acesso em 28 jun. 2018.

UN. 2000. A/55/305-S/2000/809. New York, 21 Aug. Disponível em: < http://www. un.org/documents/ga/docs/55/a55305.pdf > . Acesso em: 30 dez. 2017.

UN. 2003. Handbook on United Nations Multidimensional Peacekeeping Operations. New York: Peacekeeping Best Practices Unit/DPKO.

UN. 2008. United Nations Peacekeeping Operations Principles and Guidelines. New York: DPKO.

UN. 2012. S/RES/2085. New York, 20 Dec. Disponível em: < http://unscr.com/en/ resolutions/2085 > . Acesso em: 28 jul. 2020.

UN. 2013a. S/2013/44. New York, 22 Jan. Disponível em: < https://undocs.org/ S/2013/44 > . Acesso em: 19 mai. 2019.

UN. 2013b. S/RES/2098. New York, 28 Mar. Disponível em: < https://undocs.org/en/S/ RES/2098(2013) > . Acesso em: 19 mai. 2019.

UN. 2013c. S/RES/2100. New York, 25 Apr. Disponível em: < http://unscr.com/en/ resolutions $/ 2100>$. Acesso em: 28 jul. 2020.

UN. 2014a. A/68/19. Report of the Special Committee on Peacekeeping Operations. New York. Disponível em: < https://www.securitycouncilreport.org/atf/cf/\% 7B65B FCF9B-6D27-4E9C-8CD3-CF6E4FF96FF9\%7D/a_68_19.pdf > . Acesso em 28 jun. 2018. UN. 2014b. Final Report of the Expert Panel on Technology and Innovation in UN Peacekeeping. New York, 22 Dec. Disponível em: < https://peacekeeping.un.org/en/ 
final-report-of-expert-panel-technology-and-innovation-un-peacekeeping > . Acesso em: 28 jul. 2020.

UN.2014c. Request for Expression of Interest (EOI). Procurement Division. New York.

UN. 2014d. S/2014/692. Report of the Secretary-General on the situation in Mali. New York, 22 Sep. Disponível em: < https://minusma.unmissions.org/sites/default/ files/ n1453676_eng.pdf > . Acesso em: 30 jul. 2018.

UN. 2015a. A/70/579/Add.1. Implementation of the recommendations of the Special Committee on Peacekeeping Operations. New York, 30 Nov.

UN. 2015b. A/70/95-S/2015/446. Report of the High-level Independent Panel on Peace Operations on uniting our strengths for peace: politics, partnership and people. New York, 17 Jun. Disponível em: < https://peacekeeping.un.org/en/report-ofindependent-high-level-panel-peace-operations > . Acesso em: 28 jul. 2020.

UN. 2015c. S/2015/1030. Report of the Secretary-General on the situation in Mali. New York, 24 Dec. Disponível em: < https://minusma.unmissions.org/sites/default/ files/ n1543528.pdf $>$. Acesso em: 30 jul. 2018.

UN. 2015d. S/2015/219. Report of the Secretary-General on the situation in Mali. New York, 27 Mar. Disponível em: < https://minusma.unmissions.org/sites/default/ files/ n1508173.pdf > . Acesso em: 30 jul. 2018.

UN. 2016a. S/2016/281. Report of the Secretary-General on the situation in Mali. New York, 28 Mar. Disponível em: < https://minusma.unmissions.org/sites/default/files/ 160328_sg_report_mali_english.pdf >. Acesso em: 30 jul. 2018.

UN. 2016b. S/2016/819. Report of the Secretary-General on the situation in Mali. New York, 29 Sep. Disponível em: < https://minusma.unmissions.org/sites/default/files/ n1629765.pdf > . Acesso em: 30 jul. 2018.

UN. 2018a. Fatalities by Year, Mission and Incident Type. Disponível em: < https://peace keeping.un.org/sites/default/files/statsbyyearmissionincidenttype_5a_14.pdf > . Acesso em: 02 out. 2018.

UN. 2018b. Fatalities by Mission and Incident Type. Disponível em: < https://peacekeeping. un.org/sites/default/files/statsbyyear_1_15.pdf >. Acesso em: 02 out. 2018.

UN. 2018c. S/2018/273. Report of the Secretary-General on the situation in Mali. New York. 29 Mar. Disponível em: < https://minusma.unmissions.org/sites/default/ files/ report_of_the_secretary-general_on_the_situation_in_mali_-_29_march_.pdf > . Acesso em: 30 jul. 2018.

UN. 2018d. S/RES/2423. New York, 28 Jun. Disponível em: < https://minusma.unmissions. org/sites/default/files/n1820250_0.pdf > . Acesso em: 30 jul. 2018.

UN. 2019. United Nations Use of Unmanned Aircraft Systems (UAS) Capabilities. New York: DPO/DOS. 
Valentino, Benjamin, Huth, Paul e Balch-Lindsay, Dylan. 2004. “'Draining the sea': Mass killing and guerrilla warfare”. International Organization 58, no. 2: 375-407.

"What is the Difference Between a UAV and UAS?" 2016. DARTdrones. Disponível em: < https://www.dartdrones.com/difference-between-uav-and-uas/ > . Acesso em: 28 jul. 2020.

West, Jonathan P. e Bowman, James S. 2016. "The Domestic Use of Drones: An Ethical Analysis of Surveillance Issues". Public Administration Review 76, no. 4: 649-659.

Wood, Reed M., Kathman, Jacob D. e Gent, Stephen E. 2012. “Armed intervention and civilian victimization in intrastate conflicts”. Journal of Peace Research 49, no. 5: 647-660.

World Bank. 2006. "Enhancing Agricultural Innovation: How to Go Beyond the Strengthening of Research Systems”. Washington.

Wylie Jr, Joseph Caldwell. 1989. Military Strategy: A General Theory of Power Control, Naval Institute Press. 